\title{
The Constrained Multinomial Logit: A semi-compensatory choice model
}

\author{
F. Martinez (University of Chile) \\ F. Aguila (University of Chile) \\ R. Hurtubia (TRANSP-OR EPFL)
}

August 28, 2008 


\section{Introduction}

Traditional logit models assume a compensatory utility function (trade-off between attributes).

This approach fails to recognize attribute thresholds in consumer behavior.

A mixed strategy is proposed, using compensatory utilities with cutoff factors that that restrain choices to the available domain. 


\section{Contents of presentation}

Discrete choice problem

Constrained random utility

Constrained multinomial logit

Application examples

Calibration issues

Conclusions 


\section{The constrained discrete choice problem}

Consumer's problem:

$$
\begin{aligned}
& \max _{\delta_{n i}} \sum_{i \in C} \delta_{n i} U_{n}\left(X_{i}, p_{i}\right) \\
& \text { s.a } \sum_{i \in C} \delta_{n i}=1, \quad \delta_{n i} \in\{0,1\} \quad \forall i \in C
\end{aligned}
$$

Requires to:

Specify a utility function able to include constraints or Specify a predefined set of available alternatives $(C)$ 


\section{The constrained discrete choice problem}

Rational behavior: Max utility s.t. constraints:

$$
\begin{aligned}
\max _{\delta_{n i}} & \sum_{i \in C} \delta_{n i} U_{n}\left(X_{i}, p_{i}\right) \\
\text { s.t } & \sum_{i \in C} \delta_{n i}=1, \quad \delta_{n i} \in\{0,1\} \quad \forall i \in C \\
& a_{n k} \leq X_{i k} \leq b_{n k} \quad \forall i \in C, k=\{1, \ldots, K-1\} \\
& a_{n K} \leq p_{i} \leq b_{n K} \quad \forall i \in C
\end{aligned}
$$




\title{
Approaches
}

- Non compensatory utility, e.g. elimination by aspects

Tversky, 1972

Manski, 77

- Two stage approach.

- Generate each consumer's feasible choice set. Difficulty: large choice sets

- Heuristic to reduce choice sets

Morikawa, 95

- One step approach: reduced utility

Deterministic model: linear penalties included in utility.

Swait, 01

Continuous but non-differentiable

Simulate availability/perception implicitly in the

Cascetta - Papola, 01 extended utility. Binomial logit

Swait - BenAkiva, 87

BenAkiva - Boccara, 95

Cantillo - Ortúzar, 04

\author{
Morikawa, 95
}




\section{Constrained random utility}

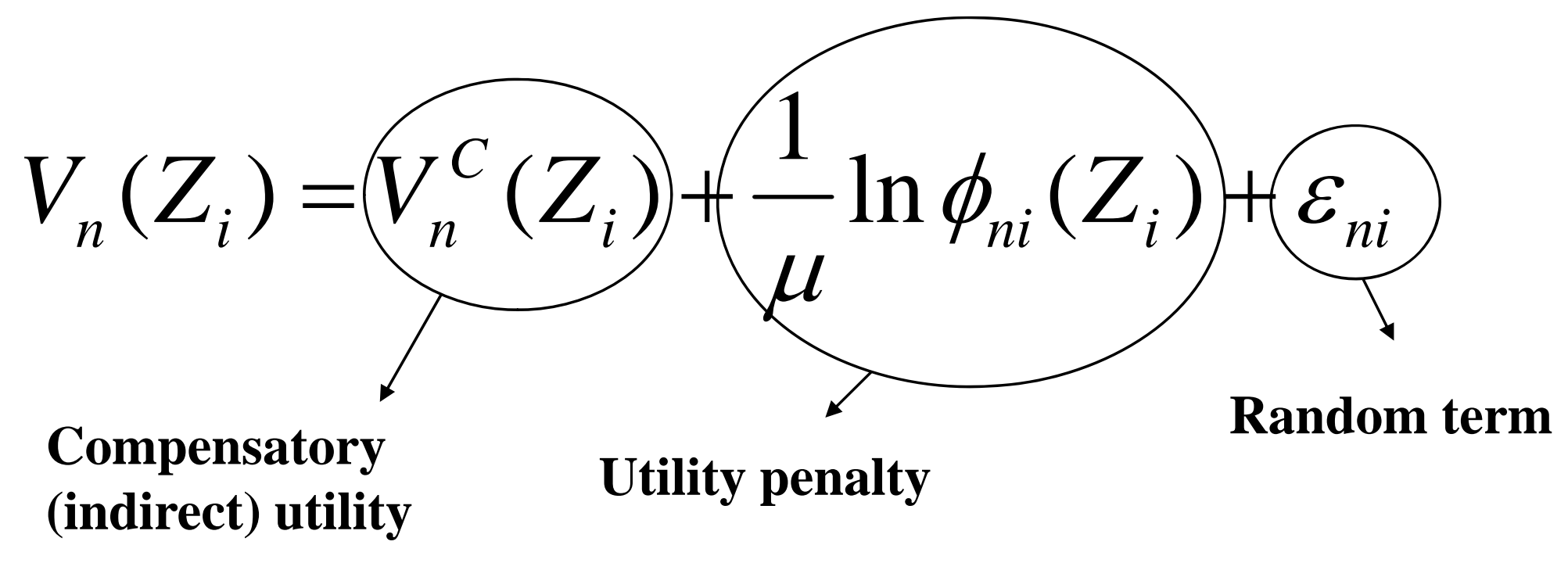

$$
\phi_{n i}=\prod_{k=1}^{K} \phi_{n k i}^{L}\left(a_{n k}\right) \cdot \phi_{n k i}^{U}\left(b_{n k}\right)
$$




\section{Constrained random utility}

Lower and upper cutoffs:

$$
\begin{gathered}
\phi_{n k i}^{L}=\frac{1}{1+\exp \left(\omega_{k}\left(a_{n k}-Z_{k i}+\rho_{k}\right)\right)}=\left\{\begin{array}{l}
\rightarrow 1 \text { if } a_{n k}<<Z_{k i} \\
\eta_{k} \text { if } a_{n k}=Z_{k i}
\end{array}\right. \\
\phi_{n k i}^{U}=\frac{1}{1+\exp \left(\omega_{k}\left(Z_{k i}-b_{n k}+\rho_{k}\right)\right)}=\left\{\begin{array}{l}
\rightarrow 1 \text { if } b_{n k}>>Z_{k i} \\
\eta_{k} \text { if } b_{n k}=Z_{k i}
\end{array}\right. \\
\rho_{k}=\frac{1}{\omega_{k}} \cdot \ln \left(\frac{1-\eta_{k}}{\eta_{k}}\right)
\end{gathered}
$$




\section{Constrained random utility}

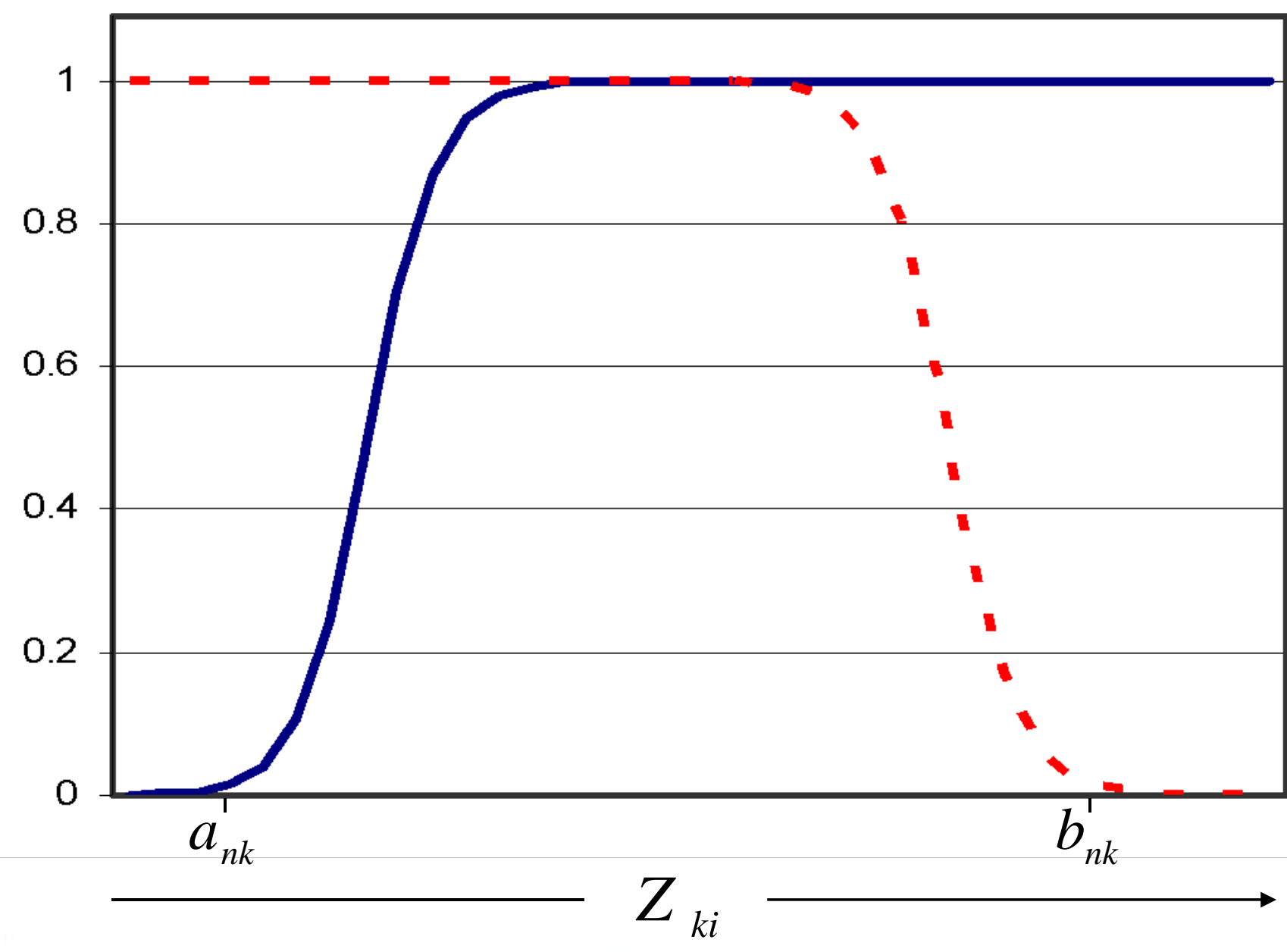

$-\phi_{n k i}^{L}$

$-\phi_{n k i}^{U}$ 


\section{Constrained Multinomial Logit}

$$
V_{n}\left(Z_{i}\right)=V_{n}^{C}\left(Z_{i}\right)+\frac{1}{\mu} \ln \phi_{n i}\left(Z_{i}\right)+\varepsilon_{n i}
$$

$$
P_{n i}=\frac{\phi_{n i} \cdot \exp \left(\mu V_{n i}^{C}\right)}{\sum_{j \in C} \phi_{n j} \cdot \exp \left(\mu V_{n j}^{C}\right)}
$$




\section{Constrained Multinomial Logit}

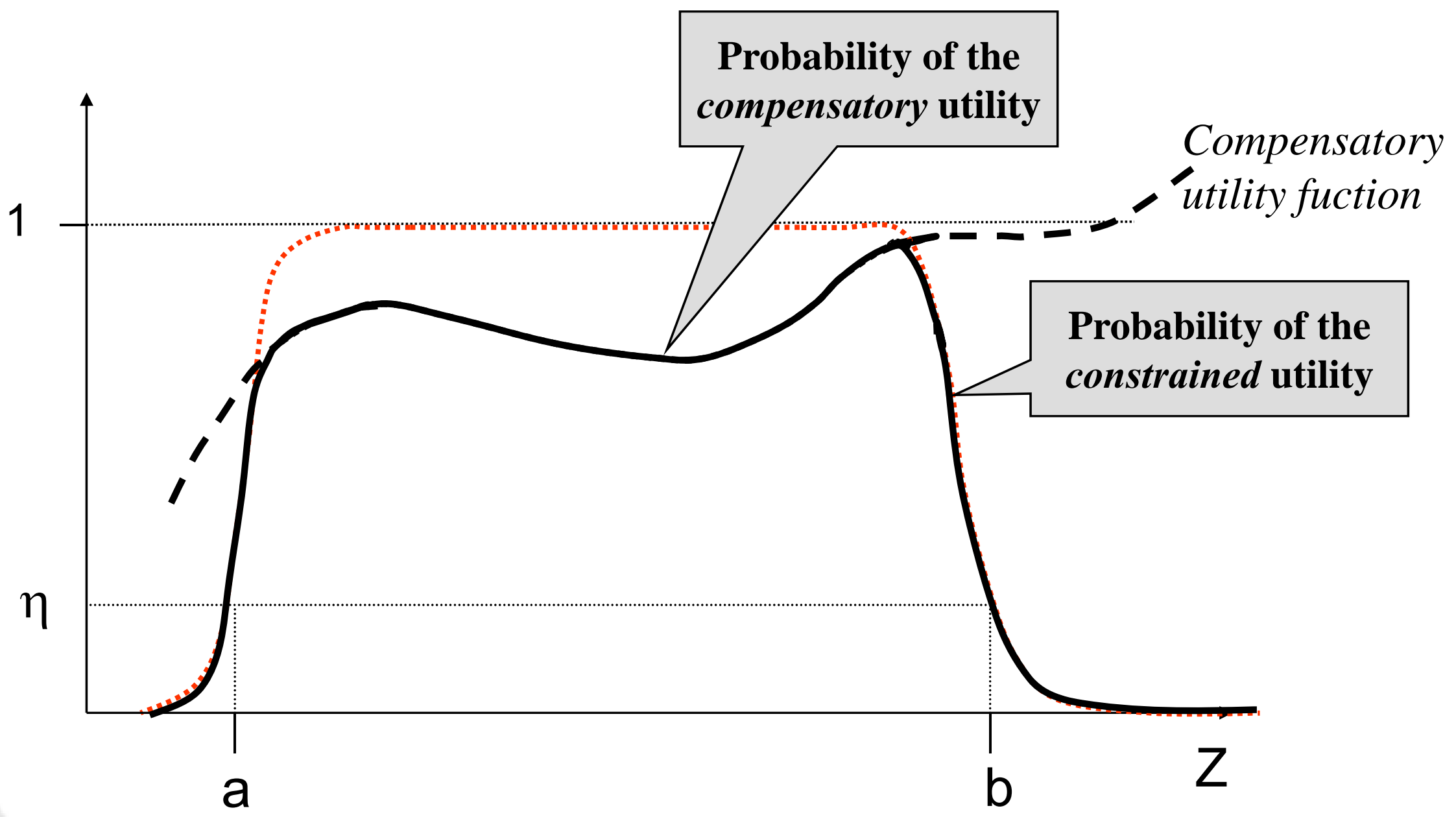




\section{Constrained Multinomial Logit}

Properties:

Preserves the closed logit formula

Represents a joint logit model

Modeling compensatory choice

Modeling constraint violation

Applications:

Real estate supply: planning regulations

Consumers: income and time budgets, attribute perception, externalities and agglomeration economies

Transport: congestion 


\section{Application example 1}

Land-use model (Martinez et al, 2008):

$$
P_{n / i}=\frac{\phi_{n i} \cdot \exp \left(B_{n i}\right)}{\sum_{g \in C} \phi_{g i} \cdot \exp \left(B_{g i}\right)} \quad B_{n i}=\alpha_{n}+\sum_{k} \beta_{n k} x_{i k}
$$$$
\phi_{n i}=\frac{1}{1+\exp \left(\omega\left(\delta_{n}-I_{i}\right)\right)}
$$

Average income in zone $i$

To estimate:

$$
\begin{aligned}
& \delta_{n}=a_{n}+\rho_{n} \\
& \omega, \beta_{n k}
\end{aligned}
$$




\section{Application example 1}

Calibration of cutoff parameters for a land use model

\begin{tabular}{|c|c|c|c|}
\hline Parameter & Income group & MNL. $\phi=1$ & CMNL $\phi \neq 1$ \\
\hline$\alpha_{n}$ & $\begin{array}{l}2 \\
3 \\
4 \\
5\end{array}$ & $\begin{array}{l}-3.329 \\
-8.130 \\
-14.228 \\
-24.808\end{array}$ & $\begin{array}{l}-0.238^{*} \\
-2.272^{*} \\
-4.781 \\
-10.257\end{array}$ \\
\hline In(floor space) & $\begin{array}{l}2 \\
3 \\
4 \\
5\end{array}$ & $\begin{array}{l}-1.840 \\
-0.078^{*} \\
-0.857 \\
0.346^{* *}\end{array}$ & $\begin{array}{l}-0.012^{* *} \\
0.493^{* *} \\
1.022 \\
2.502\end{array}$ \\
\hline $\ln$ (zone income) & $\begin{array}{l}2 \\
3 \\
4 \\
5\end{array}$ & $\begin{array}{l}0.723^{\circ} \\
1.075 \\
2.013 \\
2.442\end{array}$ & \\
\hline Accessibility & $\begin{array}{l}1 \\
2 \\
3 \\
4 \\
5\end{array}$ & $\begin{array}{l}0.283^{*} \\
1.636 \\
2.262 \\
3.926 \\
3.125^{\circ}\end{array}$ & $\begin{array}{l}0.492^{*} \\
1.692 \\
2.295 \\
3.966 \\
3.377\end{array}$ \\
\hline$\omega$ & $\begin{array}{l}1 \\
2 \\
3 \\
4 \\
5\end{array}$ & & $\begin{array}{l}-63.769^{* *} \\
-23.953 \\
-18.290 \\
-11.601^{* *} \\
-0.069^{*} \\
0.242\end{array}$ \\
\hline $\begin{array}{l}\text { Log-likelihood } \\
\text { Nr observations }\end{array}$ & & $\begin{array}{l}-3.313 \\
600\end{array}$ & $\begin{array}{l}-3.316 \\
600\end{array}$ \\
\hline
\end{tabular}

Note: Estimates without asterix are significant (t-test $>1.96)$, except when indicated by ${ }^{*}\left(1.7<\right.$ test-t<1.96) and by ${ }^{* *}$ (t-test<1.7). 


\section{Application example 1}

Similar log-likelihood

Cutoff parameters were possible to identify

Constants are lower in the CMNL (behavior explained by cutoffs)

Different forecasting results when constrained attribute changes significantly 


\section{Application example 2}

Land-use model (MUSSA 2008)

$$
\begin{aligned}
& \longrightarrow \begin{array}{l}
\text { Number of bidding } \\
\text { households }
\end{array} \\
& P_{n / i}=\frac{\phi_{n i} \cdot H_{n} \cdot \exp \left(B_{n i}\right)}{\sum_{g \in C} \phi_{g i} \cdot H_{g} \cdot \exp \left(B_{g i}\right)} \\
& \phi_{n i}=\frac{1}{\left.1+\left(\frac{1-\eta}{\eta}\right) \exp \omega\left(a_{n}-I_{i}\right)\right)} \\
& \rho=\frac{1}{\omega} \cdot \ln \left(\frac{1-\eta}{\eta}\right)
\end{aligned}
$$

To estimate 


\section{Application example 2}

\begin{tabular}{|l|r|r|r|r|r|}
\hline \multirow{2}{*}{\multicolumn{1}{c|}{ Parameter }} & \multicolumn{6}{|c|}{ Income level } \\
\cline { 2 - 6 } & $\mathbf{1}$ & $\mathbf{2}$ & \multicolumn{1}{c|}{$\mathbf{3}$} & \multicolumn{1}{c|}{$\mathbf{4}$} & \multicolumn{1}{c|}{$\mathbf{5}$} \\
\hline constant & 0 & 0.658 & -1.492 & -3.799 & -10.828 \\
\hline 1_appartment & 0 & -0.118 & 2.842 & -0.102 & -0.186 \\
\hline ln(floor space) & 0 & -0.234 & 0.356 & 1.164 & 0.047 \\
\hline \% non_res_surface & 0.816 & 2.519 & 0.444 & 2.559 & 0 \\
\hline accessibility & 1.071 & 1.288 & 1.736 & 2.875 & 2.570 \\
\hline$a_{n}$ & 3.542 & 8.630 & 11.879 & 16.610 & 24.553 \\
\hline$\omega$ & 0.3239 & 0.3239 & 0.3239 & 0.3239 & 0.3239 \\
\hline $\begin{array}{l}\text { Min tolerated average } \\
\text { zone-income (US\$) }\end{array}$ & 113 & 276 & 380 & 531 & 786 \\
\hline
\end{tabular}

\begin{tabular}{|l|r|r|r|r|r|}
\hline Income levels (US\$) & $<296$ & $296-593$ & $593-1186$ & $1186-2371$ & $>2371$ \\
\hline
\end{tabular}




\section{Application example 2}

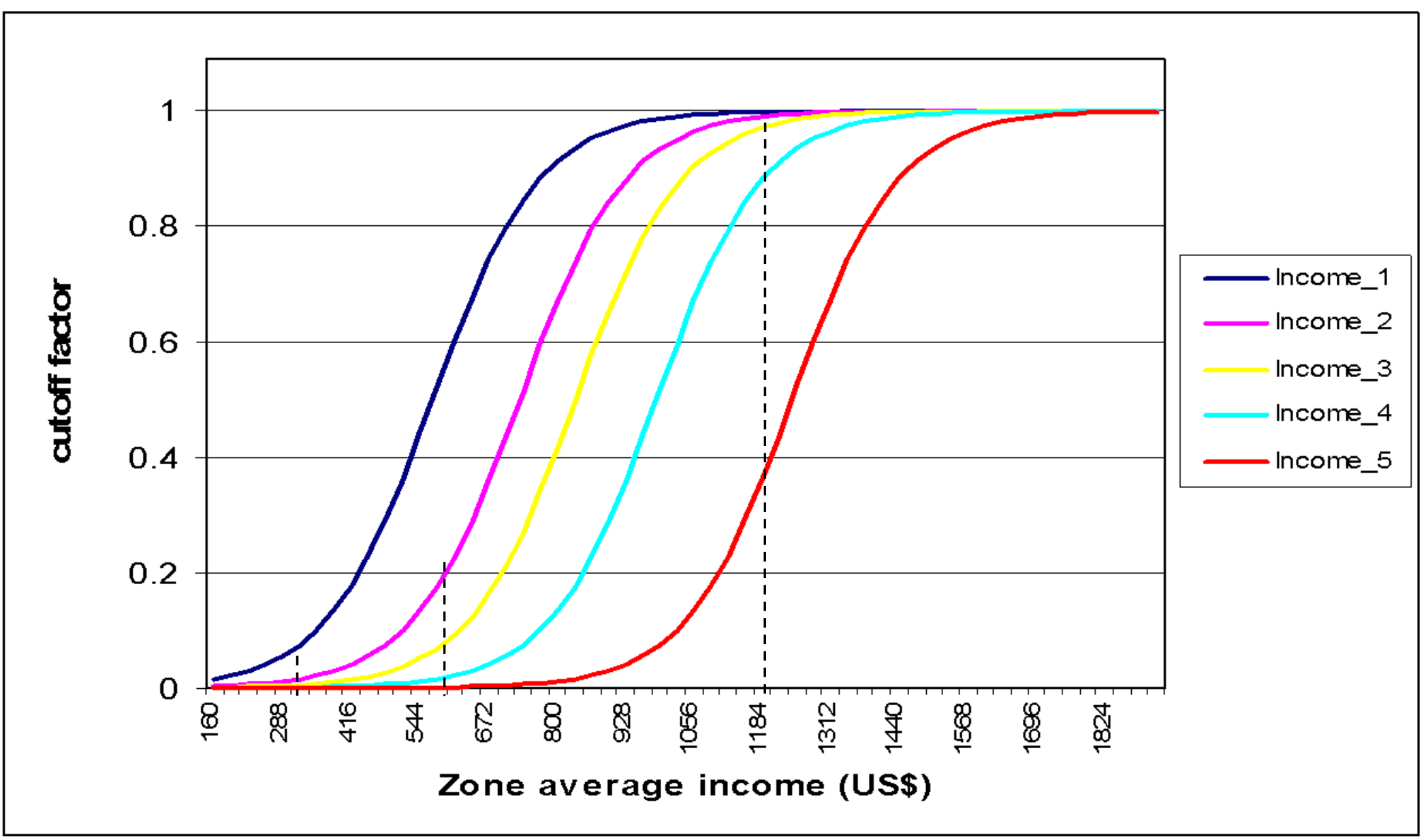




\section{Calibration issues}

Explicit exogenous constraints (budget, capacity) are useful when forecasting demand.

\section{Problems:}

- Every observation complying with restrictions.

- Correlation between parameters in the cutoff and the compensatory utility function. 


\section{Conclusions}

- The CMNL enhances the discrete choice models by imposing a realistic domain avoiding the choice set generation.

- Preserves the closed logit formula.

- Allows to include multiple constraints

- It can be used to model both endogenous and exogenous constraints.

- Requires further research on calibration methods 


\section{Questions?}

